\title{
Computer aided system for segmentation and visualization of microcalcifications in digital mammograms
}

\author{
Branimir Reljin ${ }^{1}$, Zorica Milošević2 ${ }^{2}$ Tomislav Stojić ${ }^{3}$, Irini Reljin ${ }^{1}$ \\ ${ }^{1}$ Faculty of Electrical Engineering, University of Belgrade, Belgrade, Serbia \\ ${ }^{2}$ Radiology Department, Institute of Oncology, Clinical Center of Serbia, Belgrade, Serbia \\ ${ }^{3}$ Faculty of Mechanical Engineering, University of Belgrade, Belgrade, Serbia
}

\begin{abstract}
Two methods for segmentation and visualization of microcalcifications in digital or digitized mammograms are described. First method is based on modern mathematical morphology, while the second one uses the multifractal approach. In the first method, by using an appropriate combination of some morphological operations, high local contrast enhancement, followed by significant suppression of background tissue, irrespective of its radiology density, is obtained. By iterative procedure, this method highly emphasizes only small bright details, possible microcalcifications. In a multifractal approach, from initial mammogram image, a corresponding multifractal "images" are created, from which a radiologist has a freedom to change the level of segmentation. An appropriate user friendly computer aided visualization (CAV) system with embedded two methods is realized. The interactive approach enables the physician to control the level and the quality of segmentation. Suggested methods were tested through mammograms from MIAS database as a gold standard, and from clinical praxis, using digitized films and digital images from full field digital mammograph.
\end{abstract}

Keywords: mammography, microcalcifications, medical image processing, computer aided detection (CAD), mathematical morphology, multifractal analysis

\section{Introduction}

Breast cancer is a leading cause of mortality in women population in developed countries $[1,2]$. One of significant signs of possible cancerous changes are small calcium deposits in the breast tissue, usually referred as microcalcifications [3]. Those anomalies may be viewed in radiology film as small spots brighter than surrounding $[3,4]$. Unfortunately, from several reasons: microcalcifications are small sized (typically from 0.1 up to a few of millimeters), they often appear in an inhomogeneous background tissue, and the local contrast is usually low, detection of microcalcifications is a hard task, even for skilled radiologists. By digitizing radiology films and applying digital image processing algorithms, significant improvements of image analysis are possible, for instance, contrast enhancement, which hardly improves the visibility of mammographic features. Moreover, new developments in detector technology allow direct conversion

Correspondence: BD. Reljin, Faculty of Electrical Engineering, University of Belgrade, 11000 Belgrade, Bulevar Kralja

Aleksandra 73, Serbia; e-mail: reljinb@etf.rs of X-rays to electric signal, leading to full field digital mammography (FFDM) [5]. Although the cost of FFDM devices is still a hard limiting factor for wider application of these devices, this technology has significant advances in breast cancer diagnosis including lower radiation dose, reduced breast compression pressure, and better visualization of breast tissue.

Since suspicious regions in a breast tissue may be camouflaged by background tissue, and small objects can even be lost, a reliable automated computer aided detection (CAD) system [6], sometime called as computer aided diagnostic system [7], could be very useful, providing a valuable "second opinion" to a radiologist. Computer aided detection is the process of identifying potential abnormalities within a mammogram, classifying regions of a mammogram as positive (belonging to an anomaly) or negative. The CAD system assists the radiologist by confirming the detection of suspicious regions or identifying those that might otherwise have been missed.

After processing and analyzing digital image, CAD system usually marks suspicious regions in the mammogram. Two different types of marks typically are used: asterisks $(*)$ indicating masses, and triangles $(\Delta)$ 
indicating microcalcifications (although these marks could be different, depending on the manufacturer). Radiologists review a mammogram then activate the CAD software and re-evaluate the marked area(s) before issuing a final report.

Different methods for image enhancement are suggested and embedded into CAD systems as reviewed in [8]. Note that conventional contrast enhancement algorithms and thresholding [9] are not quite appropriate since they globally change the whole image, not only particular details of interest, like microcalcifications. A number of methods have been proposed for segmentation and/or detection of microcalcifications, such as classical image filtering [10,11], techniques based on mathematical morphology $[12,13]$, stochastic fractal models [14,15], wavelet analysis [16-20] and multiscale analysis [21].

In this paper the system providing better visualization of microcalcifications, helping thus the physicians to detect brest cancer in an earlier stage, is described. System is tested over referent MIAS database [22] and applied to a number of cases from clinical praxis in the Clinical Center of Serbia [23].

\section{Materials and methods}

Microcalcifications have several characteristic features [3]. They are: (i) small bright parts, (ii) not belonging to background tissue (rare events), with (iii) relatively high local contrast, (iv) usually clustered. From these features we derived two methods for segmentation of microcalcifications. One method is based on mathematical morphology (MM) while the second one is based on the multifractal (MF) analysis.

The mathematical morphology techniques relate to modern mathematical set theory [24]. In image processing, morphological operations have been originally developed for the analysis of binary (black and white) images, and later on extended to monochrome (gray-scale) and multicomponent (color) images [9]. MM image processing use the two sets in a matrix form: an input image, $\mathbf{I}$, sized $M \times N$, described by its pixels $I(i, j), i=1,2, \ldots, M, j=1,2, \ldots, N$, and a processing operator, so-called the structuring element, $\mathbf{S}$, with elements $S(k, l)$, usually in a square form, $k=1,2, . ., K$, $l=1,2, \ldots, K$, and much smaller than the input image. Although the elements of structuring element may have arbitrary magnitudes, the most commonly used is the flat structuring element, having the same values of all nonzero elements.

Two morphological operations: erosion and dilation are fundamental to morphological processing [9]. The erosion of $\mathbf{I}$ by a flat structuring element $\mathbf{S}$ is defined as the minimum value of the image in the region coincident with $\mathbf{S}$, within the image $\mathbf{I}$. Oppositely, the dilation is defined as the maximum value of the image in the window outlined by $S$. In this way, eroded gray-scale image will be darker than the original, because the sizes of bright parts will be reduced or even removed (depending on how their values and shapes relate to the structuring element), and the sizes of dark features will be increased. Conversely, dilated gray-scale image will be brighter than original and the intensities of the dark features will be reduced.

The next two morphological operations of interest are opening and closing. The opening of image $\mathbf{I}$ by a structuring element $\mathbf{S}$, denoted as $\mathbf{I} \circ \mathbf{S}$, is defined as erosion followed by dilation, with the same structuring element, while closing, denoted as $\mathbf{I} \bullet \mathbf{S}$, has the opposite order of the operations: dilation first and then erosion. As a consequence, with gray-scale opening one can remove bright details smaller than the structuring element used. Large details, both bright and dark, which are larger than the structuring element, remain nearly unchanged. The effects are opposite when using the closing operation: dark details will be removed if they are smaller than the structuring element.

By combining image subtraction with opening and closing, the following two transformations, so-called top-hat (TH) and bottomhat (BH) transformations, are derived. The top-hat is defined as an image I minus its opening: $\mathbf{T H}=\mathbf{I}-(\mathbf{I} \circ \mathbf{S})$, while the bottom-hat is defined as the closing of $\mathbf{I}$ minus the original image: $\mathrm{BH}=(\mathbf{I} \bullet \mathbf{S})-\mathbf{I}$. Subtracting an opened image from the original image, as TH does, yield an image, which emphasizes features removed by opening. Thus, TH transformation is an excellent tool for enhancing small bright details from a nonuniform background, and it has proved its efficiency in fingerprint segmentation applications [25]. Consequently, the BH transformation produces an effect opposite to the TH. That means, one can extract dark features from a brighter background. Note that both transformations also equalize a nonuniform background illumination.

Regarding to microcalcifications, our research was addressed to derive simply, fast, and robust algorithm for emphasize small variations in local contrast, followed by reduction of surrounding tissue texture. This is especially important in the brighter areas of mammogram (which usually correspond to dense breast tissue), where the human eye is less sensitive [9]. Local contrast enhancement with high suppression of surrounding texture can be achieved by adding the difference of $\mathbf{T H}$ and $\mathbf{B H}$ images to the original image. The obtaining image, which can be called as the contrast image, $\mathbf{C}$, is given by $\mathbf{C}=(\mathbf{T H}-\mathbf{B H})+\mathbf{I}$. Note that by the difference (TH-BH) only details brighter than surrounding and smaller than structuring element are strongly emphasized while background tissue is suppressed. In fact, the gray level of previously emphasized details is increased toward white, while the overall gray level of surrounding tissue is decreased toward black. Furthermore, if we add an original image to this difference, enhanced details become even brighter than surrounding. Consequently, the enhancement of bright details smaller than the structuring element is reinforced, and uneven background (surrounding tissue texture) is highly equalized, almost regardless of overall surrounding brightness. Furthermore, this procedure can be iteratively repeated, by using the output $\mathbf{C}(n)$ from the actual iteration, $n, n=1,2, \ldots$, as the input image for the next iteration, i.e., by substituting $\mathbf{I}(n+1)=\mathbf{C}(n)$, and repeating the described procedure. The proposed method converges rapidly. From our intensive simulations, we founded that not more than three iterations are sufficient. After such a processing, final image contains only small bright details, while uneven background tissue is equalized and suppressed. Certainly, by selecting the shape and the size of the structuring element, different parts of the initial image may be enhanced or suppressed, or even removed. In case of microcalcifications, regarding to their size (usually is up to few millimeters) and most likely shape (oval), we used disk-shaped structuring element with the corresponding diameter of up to $2.2 \mathrm{~mm}$.

Multifractal approach is an efficient way for quantitative description of complex structures, objects and phenomena. Many objects and phenomena exhibit self-similar or fractal property: a structure is assumed as made of parts similar to the whole, exactly or statistically. Artificially generated fractal structures are commonly known as deterministic (or, mathematical) fractals [26,27]. These structures are generated by using exact rules and they are characterized by exactly the same fractal dimension in whole scales, thus they are referred as monofractals. Instead, a variety of natural objects may also exhibit self-similarity but only in a statistical sense. These structures are known as random fractals. The fractal dimension of such structures varies with the observed scale, thus they are referred as multifractals [27]. Fractal and multifractal 
properties of observed structure can be quantitatively described in several ways, as reported in [27-30]. Very often, the so-called Hölder exponent, $\alpha$, is used for describing local regularity of a structure, while the distribution of this quantity, known as the multifractal spectrum, $\mathrm{f}(\alpha)$, describes the global regularity of observed structure.

Different complex structures, signals and phenomena have been successfully analyzed and described through the MF approach: for instance, image processing [29-37], medical signal processing $[28,35,38,39]$, analysis of the road traffic [40] and modern multimedia traffic [41,42], even the analysis of climate changes [43-45]. The MF analysis permits us to describe signal/structure features both from local and global points of view. For instance, high values of Hölder exponent $\alpha$ denote high local changes, and opposite for low $\alpha$. Regarding to the MF spectrum $f(\alpha)$, its low values denote rare events - isolated parts in the whole structure having particular value of $\alpha$, and opposite for high $f(\alpha)$. Moreover, the MF analysis may be performed in an inverse way: find parts in the signal/structure having particular values of $\alpha$ or $f(\alpha)$. This kind of processing may be called as an inverse MF (IMF) analysis. Regarding to images, by applying MF method, we can create an ' $\alpha$-image' - a matrix of the same dimension as initial image but filled by values of $(i, j)$ with one-by-one correspondence to image pixels $I(i, j)$. From this matrix, the MF spectrum $f(\alpha)$, also in a matrix form, $f(i, j)=f(\alpha(i, j))$, may be estimated [30],[33]. From once created $\alpha$ and $f(\alpha)$ matrices (images), we can select desired range of values $\alpha$ and/or $f(\alpha)$, extracting in this way image parts characterized by these multifractal values. Similar procedure was derived for 1D signals, as well [38].

Multifractal and inverse multifractal anlysis can be modified for enhancing microcalcifications in a breast tissue. Namely, as noted earlier, calcifications are represented by small bright spots not belonging to background tissue, usually in the form of clusters, and characterized by sharp change of local contrast just at their edges $[46,47]$. In multifractal terminology, normal human tissue is characterized by high degree of self-similarity [26-28] while the tissue anomalies are considered as structural "defects", i.e., as deviations from global regularity of the background. So, the features of calcifications are described both by high values of Hölder exponent $\alpha$ (high local changes) and low values of its distribution $f(\alpha)$ (rare events, in global sense). From the modifications derived in [37] an IMF analysis is adapted just to enhance only small contrast changes within the small regions, permitting very good detection of calcifications even in radiology dense tissues.

\section{Results}

\section{Algorithms testing over gold standard}

The two methods, described shortly in previous chapter, are tested over referent mammograms from MIAS (Mammographic Image Analysis Society) database [22], used as a gold standard. The MIAS database consists of left and right breast X-ray images for 161 patients (322 images, in total) taken from the UK National Breast Screening Programme. The images has been reviewed by a consultant radiologists and classified in the three types, such as Normal (208), Benign (63), and Malignant/Abnormal (51), from which 25 images contains microcalcifications. Anomalies have been identified and marked: the location of the anomaly and the radius of a circle, which encloses it. All images are digitized to a resolution of 50 microns and 8 bits per pixel (bpp), or 256 gray levels.
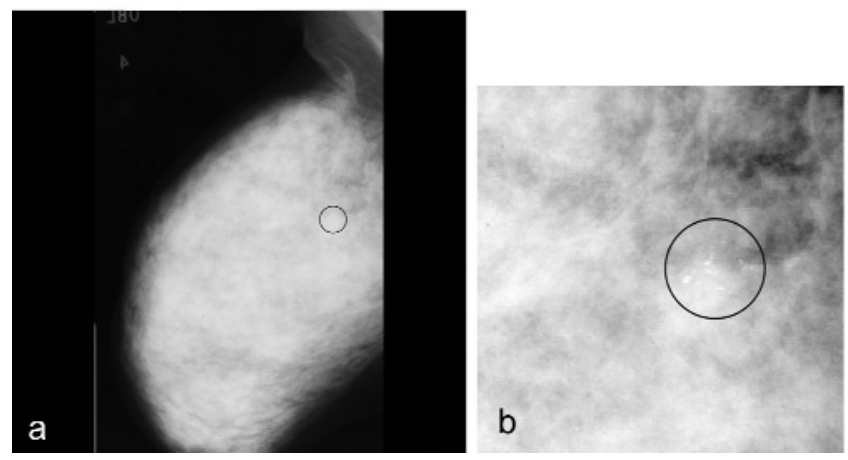

Fig. 1. (a) Original mammogram mdb253.pgm from MiniMIAS database and (b) its selected part $(256 \times 256$ pixels $)$ around the position of clinically approved microcalcifications.
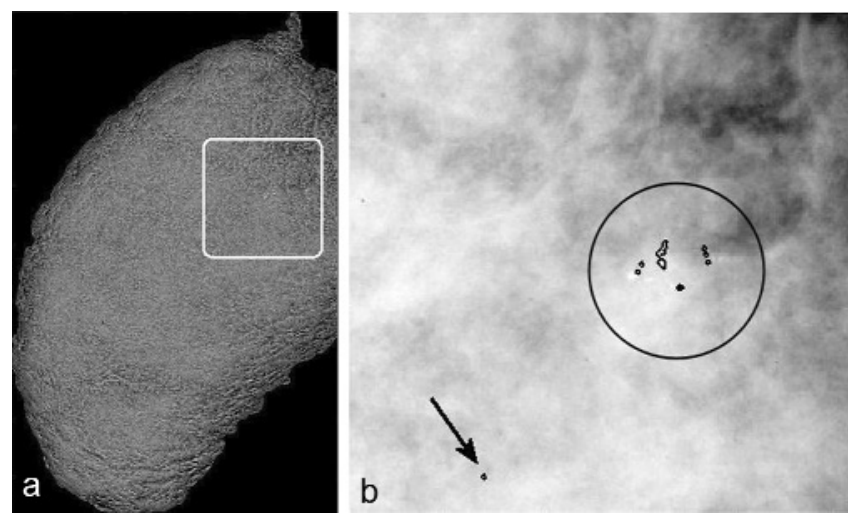

Fig. 2. Mammogram mdb253.pgm. Morphology segmentation: (a) Morphology transformed image C(3). (b) Contour lines superimposed around segmented details.

From MIAS database, a MiniMIAS database, which is publicly available at [48], was derived by rescaling the MIAS images from 50 to 200 microns (2x2 decimation). All mammograms in the MiniMIAS database have the same dimension (1024x1024 pixels) and the same 8-bit pixel depth. Regarding to pixel dimensions and the maximal size of structuring element (corresponding to $2.2 \mathrm{~mm}$ ), we used disk-shaped structuring element with maximal diameter of 11 pixels.

After applying our methods, all 25 microcalcification cases from MiniMIAS database have been detected, even in radiology hard case, such as the mammogram mdb253.pgm, depicted in Fig. 1(a). Clinically approved microcalcifications are located within a small black circle. A part of this mammogram $(256 \times 256$ pixels $)$ around approved microcalcifications is presented in Fig. 1(b). The breast tissue is very dense causing very poor contrast between anomalies and surrounding tissue. Thus, the visual detection of microcalcifications is extremely difficult even for skilled radiologists.

By applying MM method described in previous chapter, morphology transformed image after $n=3$ iter- 


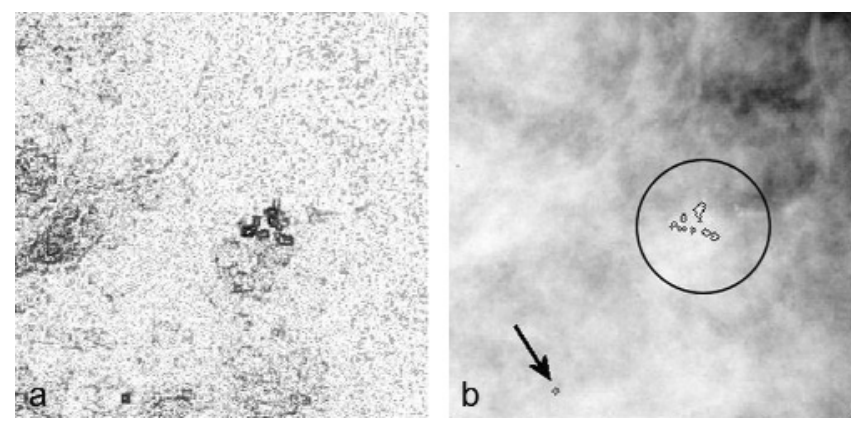

Fig. 3. Mammogram mdb253.pgm. Multifractal segmentation: (a) $f(\alpha)$ image derived from image in Fig. 1(b); (b) Contour lines superimposed around segmented regions having $0 \leq f(\alpha)<0.3$.

ations, $\mathbf{C}(3)$, of the breast region from Fig. 1(a), is shown in Fig. 2(a). Due to extremely low contrast, visual detection of the enhanced microcalcifications is still difficult. However, after applying an appropriate thresholding on the $\mathbf{C}(3)$ image, microcalcifications may be extracted and marked by contour lines, as shown in Fig 2(b).

By applying our modified IMF approach [37] on the image in Fig. 1(b), we are created corresponding $f(\alpha)$ image as in Fig. 3(a). By selecting regions within $f(\alpha)$ characterized by $0 \leq f(\alpha)<0.3$ (rare events) we can create contour lines around these regions and superimpose them over pixels from an original image, obtaining the result as in Fig. 3(b). As we can conclude, clinically approved microcalcifications are detected.

Note that besides microcalifications verified by radiologist, our methods selected also other anomalies, outside from declared region - signed by black arrows in Figs 2(b) and 3(b). By additional postprocessing, these objects may be rejected (since they are isolated objects, without any cluster), or can be further examined by a physician.

By applying both methods all microcalcification cases from MiniMIAS database (25 cases, in total) are detected as true positive $(T P)$, and no false negative $(F N)$ are detected. Furthermore, only a few false positive $(F P)$ calcifications are detected under fixed decision conditions: $F P=2$ for the MM method, with fixed number of $n=3$ iterations, and $F P=3$ for the MF method, with fixed region $0 \leq f(\alpha)<0.3$. In this way the completeness, described as $C M=T P /(T P+F N)$ was $100 \%$, and the correctness, $C R$, described as $C R=T P /(T P+F P)$, was about $90 \%$ : $92.6 \%$, for $\mathrm{MM}$ method, and $89.3 \%$, for MF method. By additional optimization of the decision algorithm, which will be the goal of our further research, it is expected to improve the correctness and thus the whole accuracy of the algorithms.

\section{Mammograms from clinical praxis}

After testing the accuracy of the MIAS database as the gold standard, we considered their applicability on different cases from medical praxis in Clinical-Hospital Center "Bezanijska Kosa", and in Institute for Oncology and Radiology from Clinical Center of Serbia. Radiologists from Clinical Center evaluated results obtained after applying our algorithms. From "Bezanijska Kosa", we used standard X-ray films $18 \times 23 \mathrm{~cm}$, digitized with 600 dpi (corresponding pixels of about 43 micrometers), obtaining images sized $4319 \times 5876$ pixels with 8bpp pixel depth, in TIFF format. From Clinical Center of Serbia we used digital images from FFDM device Hologic/Lorad Selenia [5] sized $2560 \times 3328$ pixels, $8 \mathrm{bpp}$, with 70 micrometers pixel size. Due to different pixel sizes, corresponding sizes of disk-shaped structuring elements have been rescaled to 51 and 31 pixels, respectively for digitized and digital images.

As an example, we will present here the mammogram r21.tif, Fig. 4(a), which represents cranio-caudal (CC) oblique view. Only a part of the mammogram, within the white dashed square, was analyzed. Results

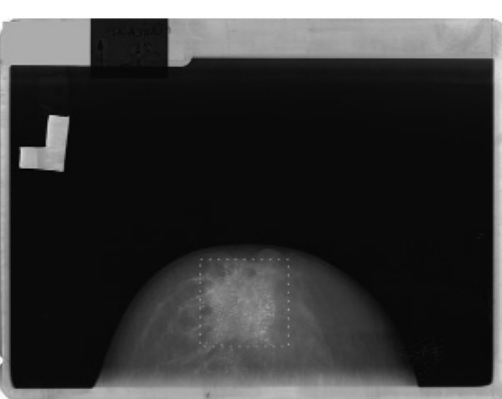

a
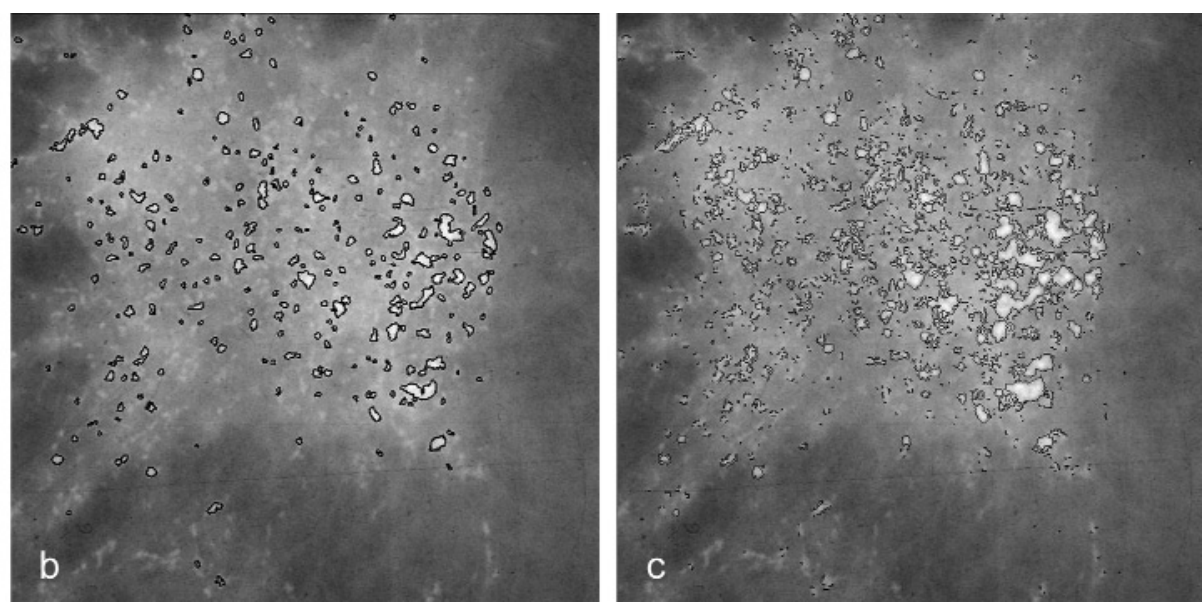

Fig. 4. (a) Mammogram r21.tif from "Bezanijska Kosa" and segmented microcalcifications in the region within the dashed rectangle, by applying: (b) the MM method and proper thresholding, (c) the MF method and selecting regions with $0 \leq f(\alpha)<0.4$ 


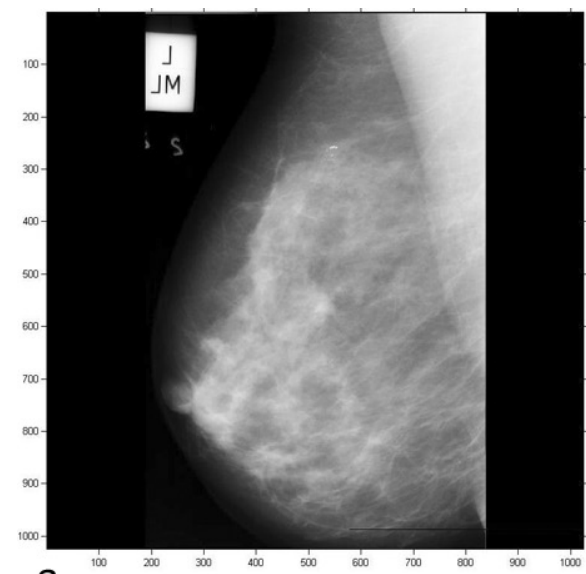

a

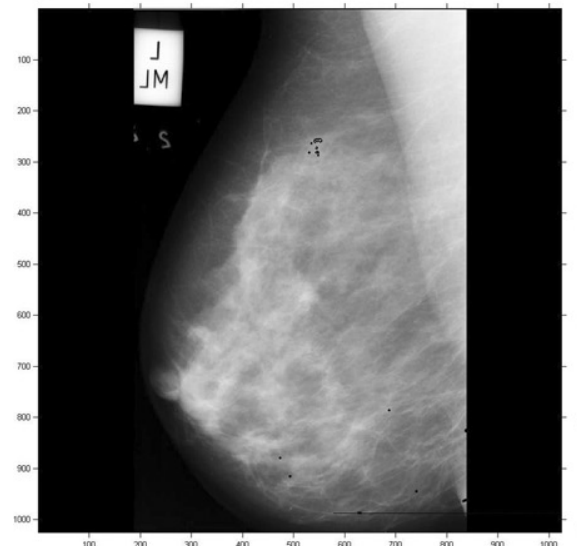

b
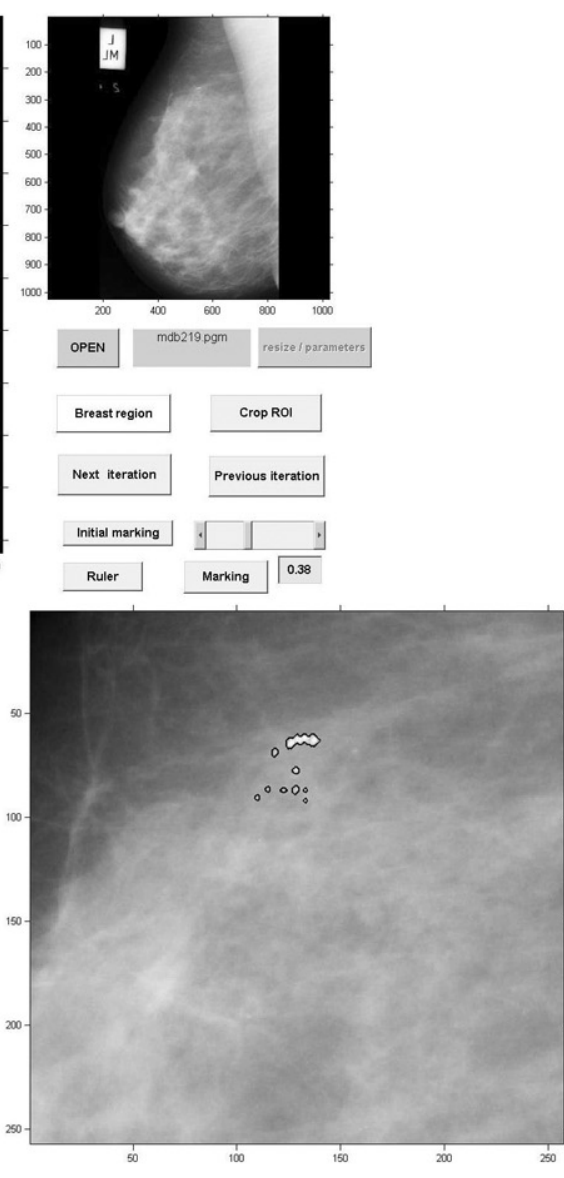

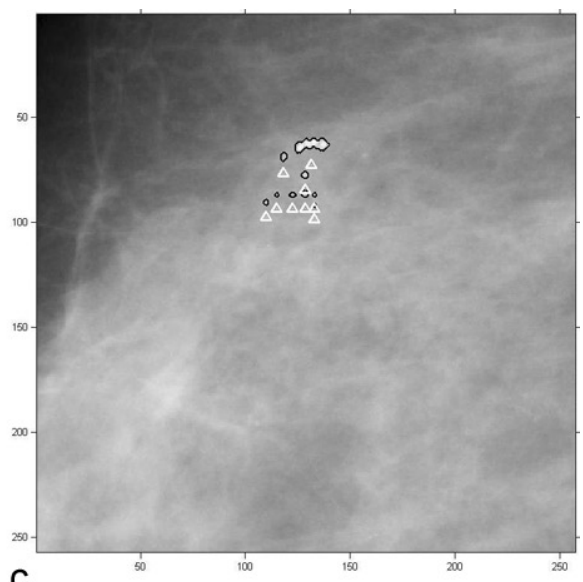

C

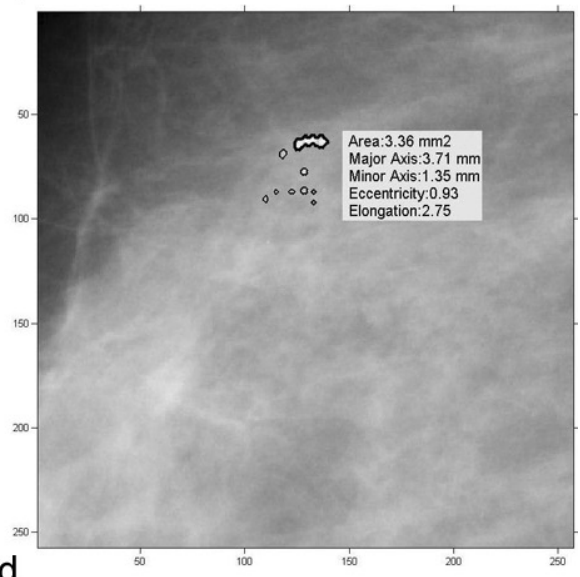

Fig. 5. (a) Our CAV system screen snapshot. Mammogram mdb219.pgm from MiniMIAS database. (b) Segmented microcalcifications with superimposed contour lines (left) and zoomed detail (right). The threshold $T=0.38$ is selected by a user. (c) Zoomed detail of segmented microcalcifications with superimposed contour lines and marked by white triangles. (d) Insertion of morphometric data describing greatest (bold outlined) selected region.

after MF and MM segmentation are depicted in Figs 4(b) and 4(c), respectively. Selected regions are confirmed by radiologists as microcalcifications.

\section{Realized CAV system}

After testing, our algorithms accompanied with necessary preprocessing steps and appropriate graphic user interface, are embedded into the CAV system, which will be briefly described. Only the MM module will be presented because the MF method has been described in details in [37].

The main screen layout of our CAV system is depicted in Fig. 5a. An original digital image (mdb219.pgm, from MiniMIAS) is on the left and the processed preview is on the right. A set of buttons on the right permits an interactive processing of an image.

By activating the button $<$ OPEN $>$ radiologist selects input mammogram from a database. If necessary, image resizing and/or the change of necessary parameters (for instance, spatial resolution and/or initial upper limit of microcalcification radius) is performed using < resize/parameters $>$ control. Before applying our MM algorithm, the mammogram is preprocessed to extract only the breast tissue region for further processing, by pressing $<$ Breast region $>$ button. Then the user can start the iterative MM procedure by activating $<$ Next iteration $>$ button. The number of iterations is arbitrary. By activating the button $<$ Previous iteration $>$ we are going back to the previous iteration step. In this way, possible overprocessing is avoided. The level of segmentation may be adjusted by changing the level of threshold $(T)$ within the normalized range $0<T<1$, by moving the slider in desired direction, or by entering the threshold value in the box below. Thresholded bright details (microcalcification candidates) will be extracted from the tissue background by activating the button $<$ Marking $>$. All pixels with a gray level $L$ larger than $T$ will be labeled and the contour lines around these objects may be created and superimposed onto the original, as shown in Fig. 5b, for the threshold of $T=0.38$. Alternatively, instead of superimposed contour lines, segmented details may be marked with white triangles, or we can use both marks, as in Fig. 5c. The thresholding procedure may be performed in an interactive way allowing adjustment of 


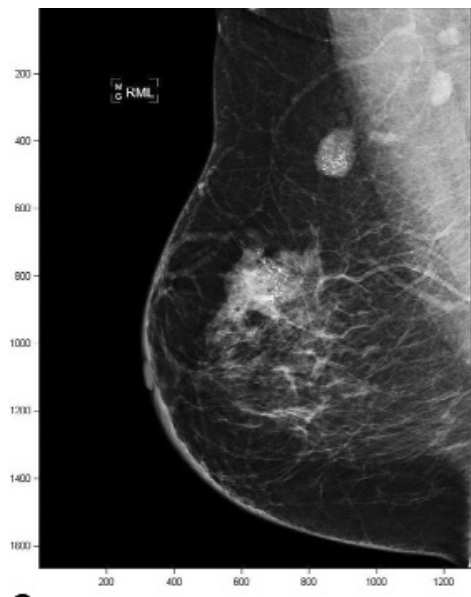

a
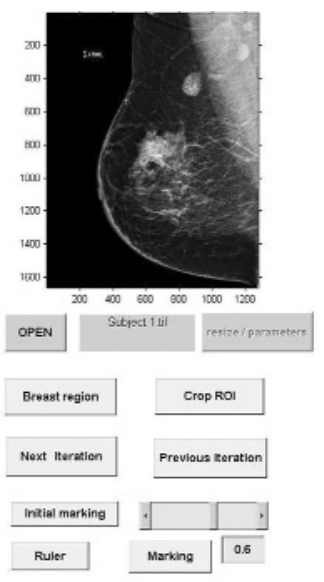
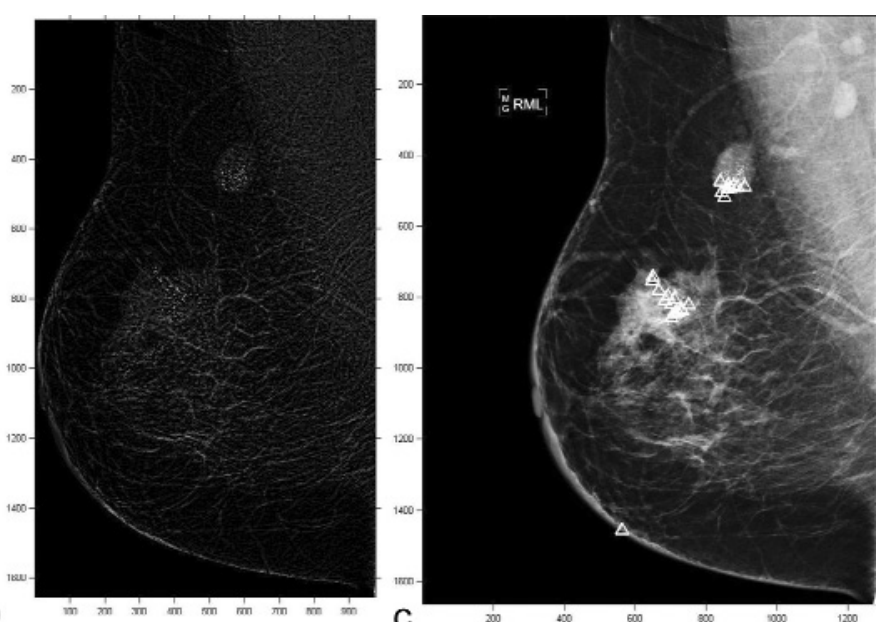

Fig. 6. Digital image Subject_1.tif - malignant case. (a) Screen layout. (b) C(3) image after MM segmentation. (c) Original mammogram with segmented details for threshold $T=0.6$, marked with white triangles.
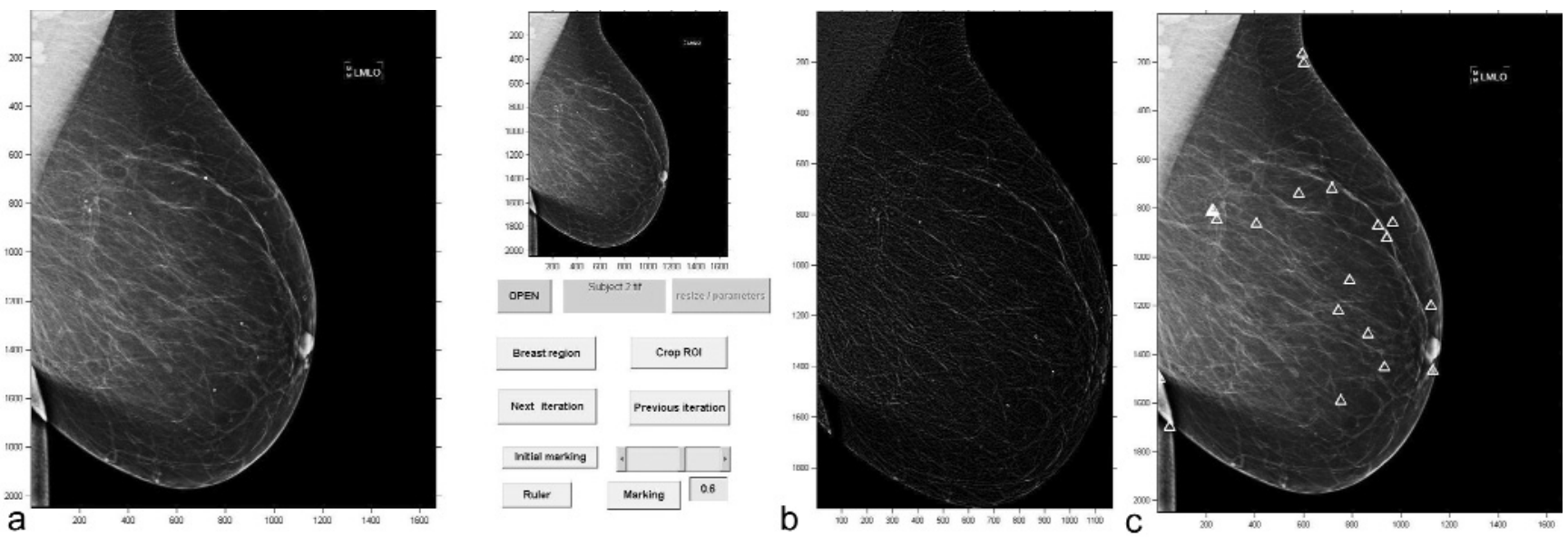

Fig. 7. Digital image Subject_2.tif - benign case. (a) Screen layout. (b) C(3) image after MM segmentation. (c) Original mammogram with segmented details for threshold $T=0.6$, marked with white triangles.

the threshold for finding the best result. Instead of interactive control, by activating $<$ Initial marking $>$ the system works with predetermined threshold value.

Control button $<$ Crop ROI $>$ allows the radiologist to interactively extract desired region of interest, and apply the algorithm only to this part, not to the whole breast region. By activating the $<$ Ruler $>$ button, different measures, for instance, distances between selected points and/or different morphometric data describing selected regions, as in Fig. 5(d), can be performed.

From a number of digital mammograms obtained from FDDM unit Hologic/Lorad Selenia [5] in Clinical Center of Serbia, only two characteristic cases are presented in Figs 6 and 7, respectively. Figs. 6(a) and 7(a) are screen snapshots of our CAV system with digital images Subject_1.tif (malignant) and Subject_2.tif (benign), while in Figs 6-7(b) and 6-7(c) MM processed $\mathbf{C}(3)$ images and images with embedded triangles, indicating to microcalciofications, are depicted. From practical reasons (faster processing), images are downsampled $2 \times 2$ times, i.e., the actual image size before processing becomes $1280 \times 1664$ pixels.

\section{Discussion}

Our research was addressed to segmentation and visualization of microcalcifications in digital mammograms. In an original image, microcalcifications are represented by small bright spots not belonging to background tissue, usually in form of clusters, and characterized by sharp change of local contrast just at their edges. In multifractal terminology, these features are described both by high values of Hölder exponent $\alpha$ (high local changes) and low values of its distribution $f(\alpha)$ (rare events in global sense). We have derived two methods for enhancing and extracting microcalcifications from the background breast tissue. One method is based on the mathematical morphology and the second on the multifractal analysis. 
From our research, it was shown that high local contrast enhancement, followed by significant suppression of surrounding tissue can be achieved using an appropriate combination of morphological top-hat and bottom-hat transformations. By a proper choice of the shape and size of structuring element, the proposed algorithm may be customized to the particular processing task. Iterative application of the proposed method highly enhances small, bright details and suppresses the background tissue. Such a procedure is suitable for mammogram analysis, since the microcalcifications, which are often an early sign of a breast cancer, are usually displayed as bright areas in a mammogram, due to their high attenuation of X-rays. From intensive simulations, it was approved that three iterations are sufficient for extracting desired details. The final segmentation is obtained by thresholding the processed image. The algorithm is fast and computationally simple being thus appropriate for real time mammogram processing and even telemedicine applications.

Multifractal analysis permits the selection of regions characterized by particular local and/or regularity. Since microcalcifications are nonregular parts of a tissue, they can be extracted as a regions characterized by low values of multifractal spectrum $f(\alpha)$. Based on this assumption an efficient MF algorithm was adapted to enhance small contrast changes permitting very good detection of calcifications even in radiology dense tissue. After obtaining multifractal 'image' $f(\alpha)$ a radiologist has the freedom to change the level of segmentation in an interactive way, by choosing appropriate range of $f(\alpha)$ values, for detecting and extracting desired regions, in our case regions which may contain microcalcifications.

Both methods are tested over MiniMIAS database as a gold standard. All microcalcification cases ( 25 in total) from this database are succesfully detected as true positive, without false negative, so the completeness, described as $C M=T P /(T P+F N)$ was $100 \%$.

The two suggested methods are embedded into the computer assisted visualization system which is used as a diagnosis confirmation tool in the clinical praxis. Note that both methods always extract small bright details which may be microcalcifications, but also other bright details including a noise, film failure and/or other artifacts. By introducing some post-processing algorithms, our CAV system may distinguish microcalcifications from other details. For instance, by applying some morphology operations we can both removes details not belonging to the tissue anomalies (for example, film emulsion artifacts) and selects and labels regions containing clusters with (possible) microcalcifications. Our further research will be addressed to the decision algorithms, for improving the accuracy of the detection of microcalcifications.
Certainly, as other CAD systems, our system is only an assistant tool, indicating to suspicious regions, and facilitating the final decision derived from the radiologist.

Acknowledgements: This work has been partially supported by the European project COST IC0604 "Telepathology Network in Europe: EURO-TELEPATH" and by the National Project of Basic Researches, no. 145096, "Automated detection of microcalcifications in digitized mammograms targeted to an early detection of a breast cancer", funded by Ministry of Science and Technology, Republic of Serbia.

\section{References}

[ 1] Borling C, Squires T, Tong T. Cancer Statistics. 1992;42(19).

[2] National Cancer Institute of Canada: Canadian Cancer Statistics. Toronto, Canada, 2004.

[3] Suri J, Setarehdan S, Singh S (Eds.), Advanced Algorithmic Approaches to Medical Image Segmentation. Springer, 2002.

[4] Bronzino J. Ed. Biomedical Engineering Handbook. CRC Press. 2000.

[5] Selenia full field digital mammography. Available at

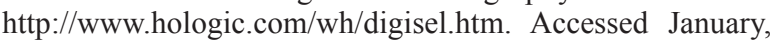
25, 2009.

[6] Yu S, Guan L. A CAD system for the automatic detection of clustered microcalcifications in digitized mammogram films. IEEE Trans. Med. Imag. 2000;19(2):115-126,

[ 7] Lee C S, Kim J K, Park H W. Computer-aided diagnostic system for breast cancer by detecting microcalcification. SPIE. 1998;3335:615-626,

[ 8] Thangavel K, Karnan M, Sivakumar R, Mohideen A K. Automatic Detection of Microcalcification in Mammograms - A Review, ICGST International Journal on Graphics, Vision and Image Processing (GVIP), Special Issue on Mammograms, 2007:23-53, C ICGST 2007, Available at http://www.icgst.com. Accessed January, 25, 2009

[ 9] Gonzalez R, Woods R. Digital Image Processing (Third Ed.). Prentice Hall, NJ. 2008.

[10] Davies D H, Dance D R. Automated computer detection of clustered calcifications in digital mammograms. Phys Med and Biol. 1990;35(8):1111-1118.

[11] Soni T, Zeidler J R, Ku W H. Performance evaluation of twodimensiomal adaptive prediction filters for detection of small objects in image data", IEEE Trans. Image Processing. 1993; 2(3):327-339.

[12] Dengler J, Behrens S, Desage J F. Segmentation of microcalcifications in mammograms. IEEE Trans Med Imag. 1993;12: 634-642.

[13] Zhao D. Rule-based morphological feature extraction of microcalcifications in mammograms. SPIE Med Imag. 1993; 1095:702-715.

[14] Lefebvre F, Benali H, Gilles R Kahn E, Di Paola R. A fractal approach to the segmentation of microcalcifications in digital mammograms. Med Phys. 1995;22(4):381-390.

[15] Li H, Liu KJR, Lo SCB. Fractal modelling and segmentation for the enhancement of microcalcifications in digital mammograms. IEEE Trans Med Imag. 1997;16(6):785-798.

[16] Brzakovic D, Brzakovic P, Neskovic M. An approach to automated screening of mammograms. SPIE Biomed Image Processing Biomed Visual. 1993;2167:868-886.

[17] Wang T C, Karayiannis N B. Detection of microcalcifications in digital mammograms using wavelets. IEEE Trans Med Imag. 1998;17(4):498-509.

[18] Lado M J, Tahoces P G, Mendez A J, Souto M, Vidal J J. A wavelet-based algorithm for detecting clustered microcalcifi- 
cations in digital mammograms. Med Phys. 1999;26(7):129412305.

[19] Bazzani A, Bevilacqua A, Bollini D. et al. Automated detection of clustered microcalcifications in digital mammograms using an SVM classifier. Proceedings of the European Symposium on Artificial Neural Networks. 2000: 195-200.

[20] Lemaur G, Drouiche K, DeConinck H. Highly Regular Wavelets for the Detection of Clustered Microcalcifications in Mammograms. IEEE Transactions on Medical Imaging. 2003;22(3):393-401.

[21] Netsch T, Peitgen HO. Scale-space signatures for the detection of clustered microcalcifications in digital mammograms. IEEE Trans Med Imag. 1999;18(9):774-786.

[22] Suckling J, Parker J, Dance DR, et al. The mammographic image analysis society digital mammogram database. Proceedings of the 2nd International Workshop on Digital Mammography. Exerpta Medica, International Congress Series. 1994;1069:375-378

[23] Milosevic Z. Quality issues in breast cancer screening, detection and diagnosis. ESO Course, Nis, Serbia, June, 2005

[24] Serra J, Image Analysis and Mathematical Morphology, Academic Press, London (UK), 1982.

[25] Mosorov V. Using tophat transformation for image fingerprints segmentation. Proc. ICSES '2001 Signals and Electronic Systems, Lodz (Poland), 2001.

[26] Mandelbrot BB. The Fractal Geometry of Nature, W. H. Freeman, Oxford, 1983.

[27] Evertsz C, Mandelbrot B. Multifractal Measures. Appendix B in Peitgen H, Jurgens H, Andrews P. Chaos and Fractals, Springer, 1992.

[28] Iannaccone P, Khokha M. (Eds). Fractal Geometry in Biological Systems, CRC Press, 1996.

[29] Véhel J L, Mignot P. Multifractal segmentation of images. Fractals. 1994;2(3):379-382.

[30] Véhel J L. Introduction to the multufractal analysis of images, Technical report INRIA, 1996.

[31] Turner MJ, Blackledge JM, Andrews PR. Fractal Geometry in Digital Imaging, Academic Press, 1998.

[32] Pesquet-Popescu B, Véhel JL. Stochastic fractal models for image processing. IEEE Signal Processing Magazine. 2002;19(5):48-62.

[33] Reljin I, Reljin B, Pavlovic I, Rakocevic I. Multifractal analysis of gray-scale images. Proc. IEEE 10th Mediterranean Electrotechnical Conference MELECON-2000; Lemesos, Cyprus, May 29-31 2000;II:490-493. Available at: http://ieeexplore.ieee.org/xpl/tocresult.jsp?isnumber $=19013$ \&isYear=2000. Accessed January, 25, 2009.

[34] Avramov Ivic M, Petrovic S D, Kalman E. et al. AFM studies of DNA structures extracted from adriamycin treated and non-treated Erlich tumor cells, $J$ Serb Chem Soc. 2005;70(6):823-831, UDC 615.33+547.963.32:616-006. Available at:

http://www.shd.org.yu/htdocs/shd/vol70/No6.html\#5. Accessed January, 25, 2009.

[35] Reljin I, Reljin B. Fractal geometry and multifractals in analyzing and processing medical data and images, Archive of Oncology. 2002; Vol.10,No.4:283-293. YU ISSN 0354-7310, UDC 616-006, Available at:

http://www.onk.ns.ac.yu/Archive/Home.asp. Accessed January, 25, 2009.

[36] Andjelkovic J, Zivic N, Reljin B, Celebic V, Salom I. Classifications of digital medical images with multifractal analysis. Proc. 8th WSEAS Int. Conf. on Signal, Speech and
Image Processing (SSIP'08). Santander, Cantabria, Spain. Sept 23-25, 2008: 88-92. ISBN 978-960-6474-008-6, ISSN 1790-5109.

[37] Stojic T, Reljin I, Reljin B. Adaptation of multifractal analysis to segmentation of microcalcifications in digital mammograms, Physica A: Statistical Mechanics and its Applications, Elsevier, 2006;367:494-508. [available online 21 December 2005, at www.elsevier.com, www.sciencedirect.com]. Accessed January, 25, 2009.

[38] Rudinac M, Uscumlic M, Rudinac S, Milovanovic B, Reljin I, Reljin B. Fractal and multifractal analysis of heart rate variability. Proc. Conf. TELSIKS'07, Nis (Serbia), Sept. 26-18. 2007;2:325-328.

[39] Reljin I, Reljin B, Avramov-Ivic M, D. et al. Multifractal analysis of the UV/VIS spectra of malignant ascites: Confirmation of the diagnostic validity of a clinically evaluated spectral analysis. Physica A: Statistical Mechanics and its Applications. Elsevier 2008;387(14):3563-3573. Available online at: http://dx.doi.org/10.1016/j.physa.2008.02.029. Accessed January, 25, 2009.

[40] Stanojevic M, Reljin I, Reljin B. Multifractal inverse processes of traffic flow variables, in Book of Abstracts 6th Balkan Conference on Operational Research, F3D.5. Thessaloniki (Greece), May 22-25, 2002:204.

[41] Reljin I, Reljin B. Neurocomputing in teletraffic: Multifractal spectrum approximation (invited paper). Proc. 5th Seminar NEUREL-2000, IEEE, Belgrade (Yugoslavia), Sept 25-27, 2000:24-31, ISBN 0-7803-55521-1, IEEE Catalog Number 00EX287, Library of Congress 99-60930.

[42] Reljin I, Samcovic A., Reljin B. H.264/AVC compressed video traces: Multifractal and fractal analysis. EURASIP Journal on Applied Signal Processing - Special Issue on Advanced Video Technologies and Applications for H.264/AVC and Beyond, 2006;20:1-13, Article ID 75217. Available at: http://www.hindawi.com/journals/asp/volume2006/si.14.html. Accessed January, 25, 2009.

[43] Reljin I, Jovanovic G, Reljin B. The climate signal analysis through linear and non-linear methods. Proc. XI ISTET'01, Linz, Austria, Aug. 19-22, 2001.

[44] Reljin I, Reljin B, Jovanovic G. Multifractal analysis of El Nino climate influence. First Balkan Conference on Signal Processing, Communications, Circuits and Systems, Balkan '2000, in Conference CD, Istanbul (Turkey), June 2-3, 2000.

[45] Jovanovic G, Reljin I, Reljin B. Multifractal analysis of El Nino influence. Proc. Conference on Non-linear Phenomena in Global Climate Dynamics, The Abdus Salam International Center for Theoretical Physics, Trieste, Italy, Sept 26-29, 2000.

[46] Bakic P R, Albert M, Brzakovic D, Maidment A D A. Mammogram synthesis using a 3D simulation, Medical Physics. 2002;29(9):2140-2151.

[47] Carton A-K, Bosmans H, Van Ongeval C, Souverijns G, Rogge F, Van Steen A, Marchal G. Development and validation of a simulation procedure to study the visibility of micro calcifications in digital mammograms, Medical Physics. 2003;30(8):2234-2240.

[48] Suckling J, The MiniMIAS database, Mammographic Image Analysis Society - MIAS. Available at: http://www.wiau.man.ac.uk/services/MIAS/MIAScom.html.

Submitted: 2 February, 2009 Accepted after reviews: 22 September, 2009 